\title{
Nontrivial relaxation dynamics of excitons in high-quality InGaAs/GaAs quantum wells
}

\author{
A. V. Trifonov, ${ }^{1}$ S. N. Korotan, ${ }^{1}$ A. S. Kurdyubov, ${ }^{1}$ I. Ya. Gerlovin,,${ }^{1}$ I. V. Ignatiev, ${ }^{1}$ Yu. P. Efimov, ${ }^{2}$ \\ S. A. Eliseev, ${ }^{2}$ V. V. Petrov, ${ }^{2}$ Yu. K. Dolgikh, ${ }^{3}$ V. V. Ovsyankin, ${ }^{3}$ and A. V. Kavokin ${ }^{1,4}$ \\ ${ }^{1}$ Spin Optics Laboratory, Saint Petersburg State University, 198504 St. Petersburg, Russia \\ ${ }^{2}$ SPbU Resource Center "Nanophotonics", Saint Petersburg State University, 198504 St. Petersburg, Russia \\ ${ }^{3}$ Departament of Physics, Saint Petersburg State University, 198504 St. Petersburg, Russia \\ ${ }^{4}$ Physics and Astronomy School, University of Southampton, Highfield, Southampton, SO171BJ, UK
}

(Dated: September 21, 2018)

\begin{abstract}
Photoluminescence (PL) and reflectivity spectra of a high-quality InGaAs/GaAs quantum well structure reveal a series of ultra-narrow peaks attributed to the quantum confined exciton states. The intensity of these peaks decreases as a function of temperature, while the linewidths demonstrate a complex and peculiar behavior. At low pumping the widths of all peaks remain quite narrow $(<0.1 \mathrm{meV})$ in the whole temperature range studied, $4-30 \mathrm{~K}$. At the stronger pumping, the linewidth first increases and than drops down with the temperature rise. Pump-probe experiments show two characteristic time scales in the exciton decay, $<10 \mathrm{ps}$ and $15-45 \mathrm{~ns}$, respectively. We interpret all these data by an interplay between the exciton recombination within the light cone, the exciton relaxation from a nonradiative reservoir to the light cone, and the thermal dissociation of the nonradiative excitons. The broadening of the low energy exciton lines is governed by the radiative recombination and scattering with reservoir excitons while for the higher energy states the linewidths are also dependent on the acoustic phonon relaxation processes.
\end{abstract}

PACS numbers: 78.67.De, 78.55.Cr, 78.47.jg, 71.35.Cc

\section{INTRODUCTION}

Excitons in two-dimensional semiconductor structures with quantum wells (QWs) and superlattices may be efficiently coupled to light because of the breaking of the wave vector selection rules along the structure growth direction. Radiative recombination rates of excitons strongly depend on their localization radii. In ideal QWs, the radiative decay of an exciton is possible only if the in-plane component of its wave vector does not exceed one of the photon to be emitted, $K_{c}=n \omega / c$, where $n$ is the refractive index, $\omega$ is the frequency of light. We note that $K_{c}=0.03 \mathrm{~nm}^{-1}$ at the exciton resonance frequency in GaAs QWs, which corresponds to an exciton kinetic energy: $E_{c}=\hbar^{2} K_{c}^{2} /\left(2 M_{X}\right)=0.06 \mathrm{meV}$, which is much lower than the characteristic thermal energy of the system at the liquid helium temperature. Here $M_{X}=0.5$ is the exciton mass in units of the free electron mass. Excitons with larger wave vectors do not interact with light. They will be referred to as nonradiative excitons. The oscillator strength of the whole exciton branch is accumulated within its little part (light cone) and the radiative decay rate can reach $10^{11} \mathrm{~s}^{-1}$ in GaAs-based structures with QWs. ${ }^{1-6}$ For nonradiative excitons, the dominant mechanism of decay is the phonon-mediated relaxation into the states with small wave vectors followed by the radiative recombination.

An important role of the reservoir of nonradiative excitons was recently recognized for semiconductor microcavities where strong light-matter coupling accelerates the exchange between radiative and nonradiative states. ${ }^{7-12}$ In conventional QW structures, all the dynamic processes are slowed down compared to microcavities, that favors considerable accumulation of nonradiative excitons. The effect of these excitons on the radiative ones may be significant. Nonradiative excitons can be created even at the strictly resonant excitation of the lowest exciton transition if the phonon energy corresponding to the lattice temperature is larger than the critical energy $E_{c} .{ }^{13}$ The in-plane component of the phonon wave vector, $q_{\|}$, is transferred to the exciton due to the conservation of momentum that results in ejection of this exciton outside the light cone.

For excitons localized by structure defects as well as for excitons scattered by free carriers, phonons and other excitons, the number of atomic oscillators contributing to the exciton oscillator strength is reduced as compared to the free exciton. The radiative decay rate for such excitons may decrease, by up to two orders of magnitude. ${ }^{14-16}$ Besides, structure imperfections may induce additional relaxation processes for radiative as well as for nonradiative excitons. In particular, the defect centers or the QW interface roughness may give rise to the considerable inhomogeneous broadening of exciton resonances and to the nonradiative exciton recombination. For this reason, many attempts were done to study the exciton dynamics in GaAs-based heterostructures of various sample quality.

The exciton dynamics was extensively studied using the photoluminescence (PL) kinetics measurements. Under nonresonant excitation, the PL pulse is characterized by a rise time, $\tau_{\text {rise }}=10-1000 \mathrm{ps}$, and a decay time, $\tau_{\text {decay }}=1-30 \mathrm{~ns}$, depending on the experimental conditions as well as on the sample design and quality. ${ }^{3,5,14,17-24}$ The theoretical analysis of the data performed in these works and also in Refs. ${ }^{2,13,15,25-27}$ al- 
lowed obtaining characteristic rates of processes occurring mainly in the reservoir of nonradiative excitons. It was found that, the characteristic time of exciton formation from free carriers lies in the range from several tens to hundreds of picoseconds. ${ }^{19,23,24,28}$ The exciton thermalization in the reservoir occurs approximately in the same time range. ${ }^{13,25,27}$ These two processes are mainly responsible for the PL rise time in high-quality structures. If radiative excitons are localized due to structure imperfections, their recombination time may become comparable with the time of exciton thermalization in the nonradiative reservoir so that it affects the rise of PL signal. ${ }^{15-17}$ The slowest exciton dynamics process is the scattering of nonradiative excitons into the light cone. This momentum relaxation process is responsible for the PL decay. A large spread of the decay time ${ }^{3,5,14,17,18,29,30}$ is possibly caused by the competition of the momentum relaxation with losses of nonradiative excitons via quenching centers in real structures.

The PL kinetics experiments did not allow to direct measuring the recombination time of radiative excitons in high-quality structures. The first attempts to measure this time at the strictly resonant excitation described in Refs. ${ }^{3,5,30,31}$ were not very successful because of the limited time resolution of the setups used. The recombination as well as the dephasing time for radiative excitons was extensively studied in the pump-probe and four-wave mixing experiments. ${ }^{32-38}$ Results of these studies also reveal large variations of the exciton recombination time in the range $1-30 \mathrm{ps}$, most probably due to the different quality of the investigated structures.

The large spread of experimental data on the exciton relaxation times in structures with QWs points out that the reliable data on characteristic rates of the relaxation processes both for radiative and for nonradiative excitons can be obtained only by the careful selection of highquality QW structures and in specific experimental conditions.

In this paper, we report on the experimental study of the dynamics of radiative and nonradiative exciton states in a specially designed high-quality heterostructure with a relatively wide InGaAs/GaAs QW of about $95 \mathrm{~nm}$ width. The peculiarity of this structure is in the presence of a set of the quantum confined exciton states, which manifest themselves as the ultra-narrow lines in the PL and reflection spectra. The PL spectra and the kinetics of pump-probe signal were measured under resonant excitation into one of the quantum confined states that allowed us to carefully control the excitation conditions. A comparative analysis of PL and reflectance spectra and of kinetics data has been performed for reliable identification of relaxation processes and extraction of their parameters. In particular, our analysis has shown that the dominant decay process for excitons at the lowest energy level is the radiative recombination for the case of low temperature and low excitation power.

We have found that the kinetics of pump-probe signal for the lowest exciton state exhibits, besides the fast component corresponding to the radiative exciton decay, a slow component, whose decay time is longer by three orders of magnitude. The long-lived component of the kinetics is caused by the relaxation of nonradiative excitons from the reservoir to the light cone. We have also identified other relaxation processes in the exciton system under study. In particular, we have found that the increase of excitation density is followed by the enhancement of the exciton-exciton and/or exciton-carrier collisions which broaden exciton lines in the PL spectra. At the same time, the temperature-activated rapid quenching of the PL intensity does not accompanied by any noticeable broadening of exciton lines. We attribute this unusual effect to the thermal dissociation of nonradiative excitons in the reservoir followed by the nonradiative losses of the excitation energy.

\section{EXPERIMENTAL DETAILS}

We have studied an InGaAs/GaAs quantum well (QW) heterostructure grown by a molecular beam epitaxy at an n-doped GaAs substrate with orientation [001]. The structure was grown at elevated temperature of the substrate of about $550{ }^{\circ} \mathrm{C}$ to prevent clusterization of Indium atoms. ${ }^{39}$ The structure contains a wide InGaAs QW layer with a nominal thickness of about $95 \mathrm{~nm}$ and the Indium content of $2 \%$. Besides, it contains a reference narrow QW $(L=2 \mathrm{~nm})$ with Indium content of $2 \%$ also. There is a gradient of the layer thicknesses of about $10 \%$ per $\mathrm{cm}$ and the Indium content varies from 1.5 to $2 \%$ in the structure so that the real thickness and potential profile of the wide QW were estimated from spectroscopic data. The sample was cooled using an optical cryostat with a close cycle of helium cooling. The sample temperature was varied in the range of $4-30 \mathrm{~K}$.

The PL was excited by radiation of a tunable continuous-wave Ti:sapphire laser. We excited the QW excitons either quasi-resonantly into one of the quantum confined exciton levels or higher in energy up to the absorption edge of GaAs barriers. The PL was dispersed and detected by a $0.55 \mathrm{~m}$ spectrometer equipped with a CCD. The spectral resolution in actual range was about $30 \mu \mathrm{eV}$. Laser spot on the sample was about $50 \mu \mathrm{m}$ and excitation power varied in range $2-150 \mu \mathrm{W}$. The PL excitation (PLE) spectra were measured using the same setup by means of continuous tuning of the laser wavelength and detecting the PL spectra in the range of several lowest exciton transitions for each excitation wavelength. We would like to note that, due to high quality of the sample, the intensity of PL from two lowest exciton levels dominated over the scattered light of laser tuned to photon energy only one meV above these levels.

Another experimental technique exploited in our study is the pump-probe method. We used pulsed radiation of a femtosecond Ti:sapphire laser, split into the pump and probe beams. The pump beam passed through an acousto-optical tunable filter, which cut out a spectrally 
narrow pulses with full width at half maximum of about $0.6 \mathrm{~nm}$ corresponding to spectrally-limited pulse duration, $T_{p u}=1.75 \mathrm{ps}$. The pump wavelength was tuned to excite predominantly the lowest one or two exciton transitions only. The probe pulses were spectrally broad and short, of about $0.1 \mathrm{ps}$. To detect the time-resolved photomodulated reflection at different exciton transitions, the spectrum of reflected probe beam was analyzed using a $0.55 \mathrm{~m}$ spectrometer equipped with a photodiode.

\section{EXPERIMENTAL RESULTS}

\section{A. PL, PLE, and reflection spectra at low temperature}

The PL and reflectance spectra taken from the same spot on the sample are shown in Fig. 1. The spectra consist of several narrow peaks (resonances), whose positions and widths coincide in both spectra with high accuracy. The absence of any detectable Stokes shift (within experimental error in a few tens of $\mu \mathrm{eV}$ ) between the peaks in the PL and reflectance spectra indicates high quality of the sample.

It is naturally to assume that the observed resonances are due to quantization of exciton motion across the QW layer. In this case, the energies of resonances can be roughly estimated using a simple model of a wide QW with infinitely high barriers, in which the exciton is quantized as a whole: ${ }^{40}$

$$
E_{X N}=E_{g}-E_{B}+\frac{\hbar}{2 M_{X}}\left(\frac{\pi N}{L^{*}}\right)^{2},
$$

where $E_{g}$ is the band gap, $E_{B}$ is the exciton binding energy (exciton Rydberg), $M_{X}$ is the exciton mass, and $L^{*}$ is the effective thickness of QW layer, which is smaller than the real width $L_{Q W}$ by the dead layer width, $\delta L_{d} \cdot{ }^{41,42}$ The dead layer width is governed by the exciton Bohr radius, $a_{B}$. In our case, $L^{*}=L_{Q W}-2 \delta L_{d} \approx 75 \mathrm{~nm}$ assuming that $\delta L_{d}=0.8 a_{B}=10 \mathrm{~nm}$ as it is estimated for QWs with a layer thickness of about $6 a_{B} \cdot{ }^{42}$ Using the above equation, we obtain the following energy positions of quantum confined states relative to the lowest one: $\left(E_{X N}-E_{X 1}\right)_{\text {calc }}=0,0.40,1.07,2.00$ (in meV) for $N=$ $1 \ldots 4$. The obtained values well agree with the energy distances between the $X N$ and $X 1$ peaks found in the experiments (see Fig. 1 and Tab. I): $\left(E_{X N}-E_{X 1}\right)_{\text {exp }}=0$, $0.50,1.12,1.88$ (in $\mathrm{meV}$ ) for $N=1 \ldots 4$.

The resonances in the PL spectrum can be well fitted by a set of Lorentzians, $L_{X N}(E)=$ $\left(A_{X N} / \pi\right) \delta E_{X N} /\left[\left(E-E_{X N}\right)^{2}+\delta E_{X N}^{2}\right]$, superimposed on a background signal. Here $\delta E_{X N}$ is the half width at half maximum (HWHM) of the Lorentzian and $A_{X N}$ is the area under the Lorentzian curve. The parameters of Lorentzians are given in Tab. I. Because of gradients of the QW width and of the Indium content, the relative $\mathrm{PL}$ intensities from different exciton levels, $A_{X N} / A_{X 1}$,

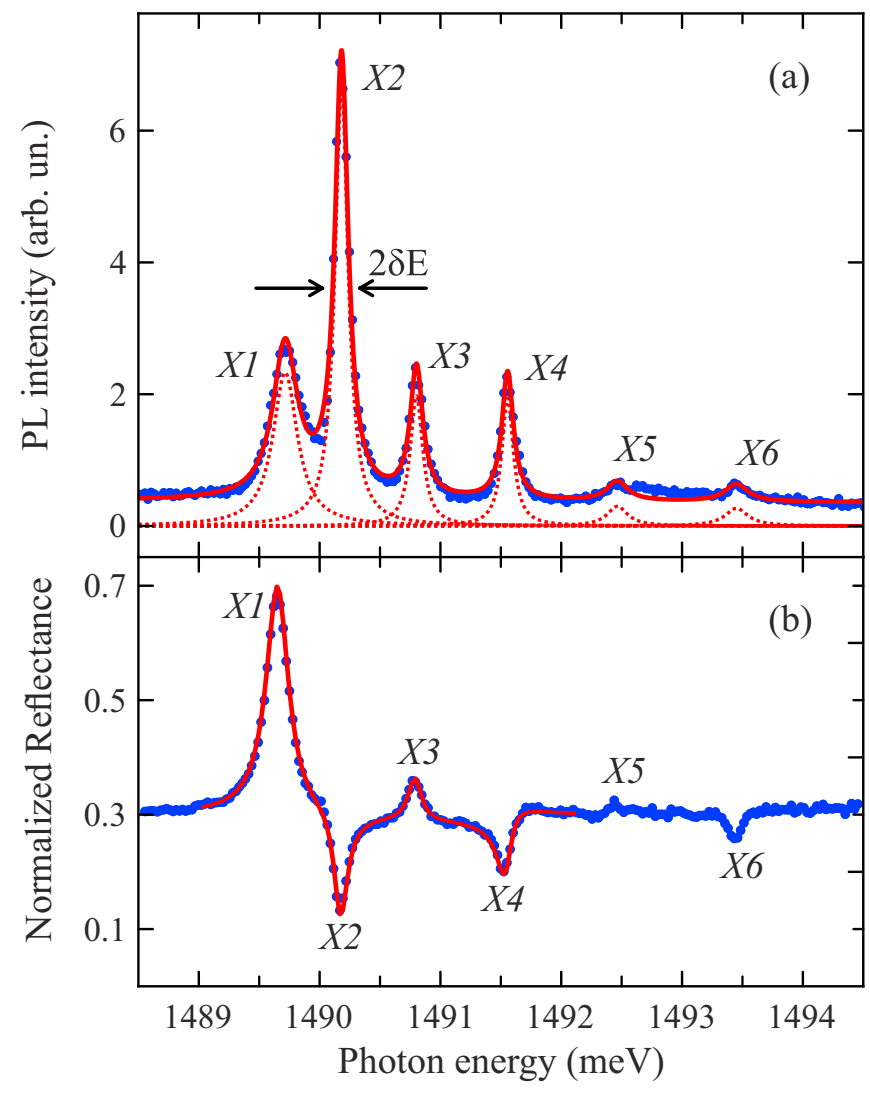

Figure 1. (Color online) (a) PL spectrum of the InGaAs/GaAs QW with nominal width $L=95 \mathrm{~nm}$ measured at temperature $T=4 \mathrm{~K}$ and excitation power $P=50 \mu \mathrm{W}$ (blue dots). The PL is excited into the $X 8$ exciton level. Thin dashed curves are the Lorentzians with parameters listed in Tab. I for the first four transitions. Notations "XN" indicate transitions from the quantum confined exciton levels. The sum of Lorentzians is shown by red solid curve. (b) Reflectance spectrum normalized to the excitation intensity (blue dots). Red solid curve shows the spectrum calculated in framework of the model described in the text with parameters listed in Tab. I.

slightly depends on the point on the structure surface. Therefore most of the measurements were done at the same spot of the sample. It should be emphasized that the PL intensity for higher exciton states is comparable with that for the lowest energy state. This feature strongly distinguishes the QW structure under study from those studied by other authors, where the highenergy exciton peaks in PL spectra are virtually absent (see, e.g., Ref. ${ }^{43}$ ).

The analysis of the PL spectrum does not provide a direct knowledge of the radiation rates because the intensity of a PL peak depends also on the exciton population, which can be different for different quantum confined states. As one can see from Tab. I, the integral intensities of the first and second exciton peaks in the PL spectrum are very similar, but this indicates only that the population of the second exciton level is significantly 
larger than that of the first one.

In a contrast to the PL spectrum, the quantitative analysis of reflectance spectrum allows one to obtain valuable information about radiative and nonradiative decay rates for different exciton states. First, we would like to note that the maximum reflectivity at the first resonance is about 0.7 , not far from unity corresponding to the totally "metallic" reflection. This is the first indication that the radiative decay of these excitons prevails over the nonradiative one.

We have performed a simple analysis of the reflectance spectrum generalizing the theory developed in Ref. ${ }^{40}$ for the case of several exciton quantum confined states. The coefficient of amplitude reflectance from a QW with several exciton resonances can be written in the form:

$$
r_{Q W}=\sum_{N=1}^{N_{\max }} \frac{i(-1)^{N-1} \Gamma_{0 N} e^{i \varphi_{N}}}{\omega_{0 N}-\omega-i\left(\Gamma_{0 N}+\Gamma_{N}\right)} .
$$

Here $\omega_{0 N}$ is the resonance frequency, $\Gamma_{0 N}$ and $\Gamma_{N}$ are the radiative and nonradiative damping rates. Phase $\varphi_{N}$ appears in this equation due to an asymmetry of the QW potential caused by the Indium segregation during the heterostructure growth process. ${ }^{44}$ Reflectance, $R(\omega)$, from the structure with a top barrier layer of thickness $L_{b}$ and a QW layer of thickness $L_{Q W}$ is calculated by a standard manner: ${ }^{40}$

$$
R(\omega)=\left|\frac{r_{01}+r_{Q W} e^{2 i \phi}}{1+r_{01} r_{Q W} e^{2 i \phi}}\right|^{2},
$$

where $r_{01}$ is the amplitude reflectance from the sample surface. Phase $\phi=K\left(L_{b}+L_{Q W} / 2\right)$ where $K$ is the photon wave vector in the heterostructure.

Result of simulation of the reflectance spectrum using Eqs. (2), (3) is shown in Fig. 1(b). Respective fitting parameters are listed in Tab. I. As seen the calculated curve very well reproduces the experimentally measured spectrum. Although the number of fitting parameters is large, they are well defined because determine different peculiarities of the resonances. In particular, ratio $\Gamma_{0 N}^{2} /\left(\Gamma_{0 N}+\Gamma_{N}\right)^{2}$ determines the peak amplitude of resonance $N$ and $\hbar\left(\Gamma_{0 N}+\Gamma_{N}\right)$ determines its HWHM. Phase $\varphi_{N}$ determines the asymmetry of resonance that is its deviation from a Lorentzian, which is clearly seen, e.g., for the $X 4$ resonance in Fig. 1(b).

The comparison of the obtained radiative and nonradiative damping rates shows that the radiative broadening is really prevail for the first exciton resonance. Real magnitudes of nonradiative broadening are even smaller than those given in Tab. I because the limited resolution of our setup (of about $30 \mu \mathrm{eV}$ ) gives rise to an additional broadening the resonances measured. We should note that the spectral broadening of the lowest transition in both the PL and reflection spectra is of about $0.1 \mathrm{meV}$ that is extremely small for structures of this type known in literature, see, e.g., Refs. ${ }^{18,30,45-47}$

We estimate the ratio of radiative decay rates of different exciton transitions using a simplified model of size
Table I. Fitting parameters of the PL and reflectance spectra.

\begin{tabular}{|c|c|c|c|c|c|}
\hline Exp. & Exciton level & $\mathrm{X} 1$ & $\mathrm{X} 2$ & $\mathrm{X} 3$ & $\mathrm{X} 4$ \\
\hline PL & A (arb. un.) & 1080 & 1390 & 390 & 420 \\
\hline PL & $\mathrm{E}(\mathrm{meV})$ & 1489.71 & 1490.18 & 1490.80 & 1491.56 \\
\hline PL & $\delta E(\mu \mathrm{eV})$ & 123 & 67 & 63 & 58 \\
\hline Refl & $\hbar \omega_{0}(\mathrm{meV})$ & 1489.65 & 1490.17 & 1490.79 & 1491.55 \\
\hline Refl & $\hbar \Gamma_{0}(\mu \mathrm{eV})$ & 47.5 & 19.5 & 6 & 11 \\
\hline Refl & $\hbar \Gamma(\mu \mathrm{eV})$ & 38.5 & 60 & 57 & 66 \\
\hline Refl & $\varphi(\mathrm{rad})$. & 0 & -0.06 & -0.03 & 0.40 \\
\hline
\end{tabular}

quantization of an exciton motion in a symmetric QW with infinitely high barriers. ${ }^{48}$ The envelope wave functions of the center-of-mass motion of the exciton as a whole are proportional to $\cos \left(N \pi z / L^{*}\right)$ for odd number $N$ of the quantum confined exciton state and to $\sin \left(N \pi z / L^{*}\right)$ for even $N$, where $z$ is the exciton center of mass coordinate along the structure axis.

The rate of the radiative transition, $\Gamma_{0 N}$, is proportional to the squared overlap integral of the exciton wave function with the light wave, $F_{N}^{2}(K)$, which, in framework of this model, is: ${ }^{48}$

$$
F_{N}^{2}(K)=\left\{\begin{array}{l}
C_{N}^{2} \cos ^{2}\left(K L^{*} / 2\right) \text { for odd } N \\
C_{N}^{2} \sin ^{2}\left(K L^{*} / 2\right) \text { for even } N
\end{array}\right.
$$

where

$$
C_{N}=\frac{N \pi / L^{*}}{\left[\left(N \pi / L^{*}\right)^{2}-K^{2}\right]} \sqrt{2 / L^{*}}
$$

Here $K$ is the photon wave vector in the QW material. As seen from Eqs. $(4,5)$, this rate may be strongly different for exciton states with even and odd numbers and gradually decreases with $N$. For the case of QW under study, equations (4) and (5) give the following radiative decay rates for different quantum confined states normalized to the rate of $X 1$ transition: $\left(\Gamma_{0 N} / \Gamma_{01}\right)_{\text {calc }}=$ $F_{N}^{2}(K) / F_{1}^{2}(K)=1,0.28,0.04,0.06$ for $N=1, \ldots 4$. Numerical calculations for QWs of similar widths in more accurate models give rise to similar results. ${ }^{42}$ The dependence of normalized rate on number $N$ of exciton state qualitatively reproduces the dependence obtained from the reflectance spectrum: $\left(\Gamma_{0 N} / \Gamma_{01}\right)_{\exp }=1,0.41,0.13$, 0.23. At the same time, the quantitative disagreement between the calculated and experimental data indicates that the exciton wave functions in real structure considerably differ from those used in the above simple model.

The exciton radiative time can be estimated from the given in Tab. I values of $\Gamma_{0 N}$ using relation: $\tau_{X N}=$ $1 /\left(2 \Gamma_{0 N}\right)$, see, e.g., Ref., ${ }^{40}$ p. 92 . For the first two transitions, the radiative times are: $\tau_{X 1}=6.9 \mathrm{ps}$ and $\tau_{X 2}=$ $17 \mathrm{ps}$. These values are of the same order of magnitude as the radiative recombination time, $\tau_{\text {rad }} \sim 10 \mathrm{ps}$, reported previously for GaAs QWs. ${ }^{3,5}$ As it seen from Tab. I, the 
nonradiative broadening $\hbar \Gamma_{X 1}$ for the first exciton state is smaller than for the higher states. This means that the broadening of excited states is contributed by additional nonradiative mechanisms.

For a deeper insight into the origin of relaxation processes in the structure, we have measured the PLE spectra of the exciton resonances. Fig. 2(a) shows the spectral dependences of integral intensity for the first four peaks on the photon energy of excitation. The spectra display several remarkable features. ${ }^{49}$ The $X_{l h}$ band was identified as the lowest state of the light hole exciton. The degree of circular polarization of PL measured at this resonance has the opposite sign compared to the polarization degree of the heavy hole resonances. This effect is due to different selection rules for respective optical transitions and is well known in literature. ${ }^{50}$ The $X_{l h}$ exciton state is split off from the $X 1$ exciton mainly due to the internal strain in the GaAs/InGaAs structure caused by the lattice mismatch of the QW and barriers.

The efficiency of excitation of all the resonances is synchronously changed with the photon energy increase up to the energy of the transition $X_{l h}$. However, above this transition, the lowest exciton state $(X 1)$ is populated more efficiently than other states, so that the relative intensity of the corresponding exciton resonance increases. This is an indication that one more relaxation mechanism is "switched on" in this spectral range of excitation. Its possible origin will be discussed in Section III.

Fig. 2(b) shows the dependence of HWHM of the first four exciton peaks on the excitation photon energy. The HWHM were obtained by Lorentzian fitting of the PL spectra measured at each excitation energy. The excitation energy was scanned with small step of about $0.05 \mathrm{meV}$. As one can see from the figure, the peak broadening noticeably increases above the $X_{l h}$ transition. A particularly strong increase of HWHM is observed for the first resonance that also indicates an additional mechanism of the broadening, which switches on at these photon excitation energies.

\section{B. Temperature and pump power dependences of the exciton resonances}

The experimental data discussed in the previous section have been obtained at low temperature $(4 \mathrm{~K})$ and low excitation power $(10-50 \mu \mathrm{W})$. In this case the phononmediated relaxation and the exciton-exciton scattering are not very efficient. To investigate the role of phononmediated processes, we have studied the temperature variations of PL spectra. The temperature rise has been found to be accompanied by a synchronous decrease of the integral amplitude of all exciton resonances, leading to the approximately 20 -fold decrease of the total PL intensity with the temperature increase from 4 to $30 \mathrm{~K}$ (see Fig. 3). The temperature dependence of the total

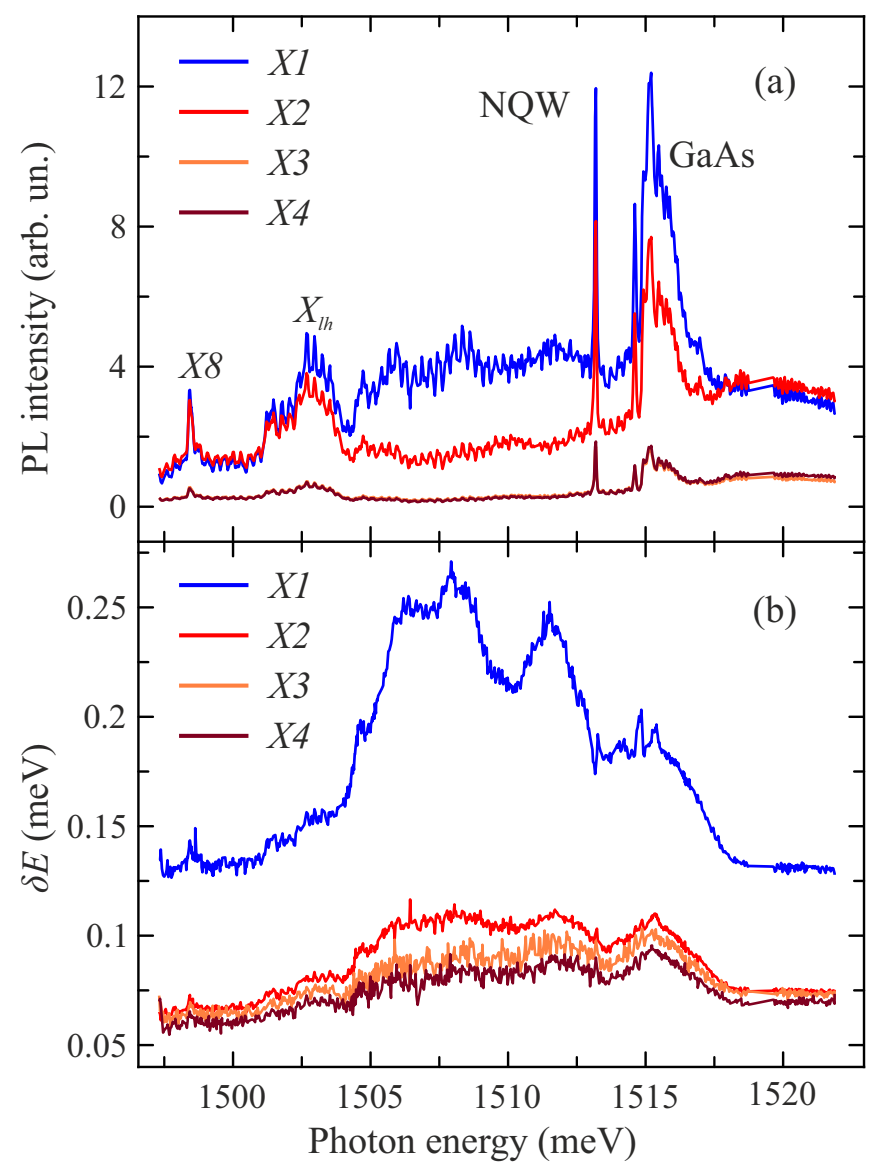

Figure 2. (Color online) PLE spectra for resonances $X 1, \ldots X 4$. Legends show identification of separate maxima: $X 8$ is the eighth quantum confined state of the heavy-hole exciton; $X_{l h}$ is the lowest state of the light-hole exciton; "NQW" is the lowest state of the heavy-hole exciton in the narrow QW; "GaAs" is the exciton state in the GaAs barriers. (b) HWHM of the resonances as functions of excitation energy. Excitation power $P=50 \mu \mathrm{W}$.

PL intensity can be well approximated by expression:

$$
I(T)=\frac{P}{1+\left(\gamma_{0 X} / \gamma_{r}\right) \exp \left(-\frac{E_{X}}{k T}\right)+\left(\gamma_{0 b} / \gamma_{r}\right) \exp \left(-\frac{E_{b}}{k T}\right)} .
$$

This expression is derived from the balance equation for the exciton population $n_{X}$ accounting for the relaxation to radiative states with rate $\gamma_{r}(\mathrm{~T})$ as well as for two processes of thermally-activated dissipation of excitons. In such conditions, the balance equation reads:

$$
\frac{d n_{X}}{d t}=P-\left[\gamma_{r}(T)+\gamma_{X}(T)+\gamma_{b}(T)\right] n_{X} .
$$

Here $P$ is the rate of optical excitation. A theoretical analysis shows ${ }^{4,14,51}$ that the exciton relaxation time, $1 / \gamma_{r}(T)$, in QWs is proportional to the sample temperature. The temperature dependences of the exciton dissipation rates are described by Boltzmann functions:

$$
\gamma_{i}(T)=\gamma_{0 i} \exp \left(-E_{i} / k T\right)
$$




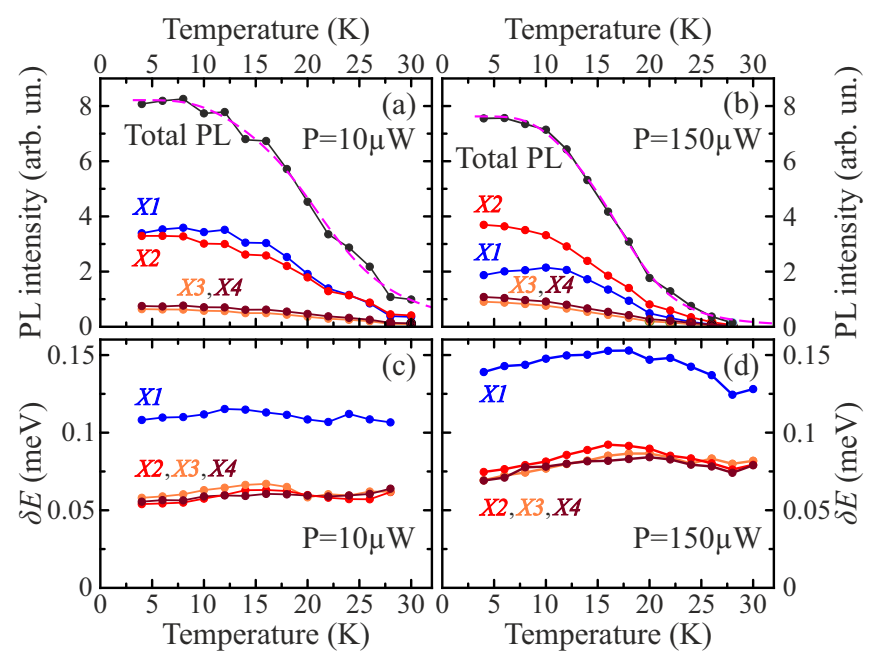

Figure 3. (Color online) Temperature dependencies of integral PL intensities ( $\mathrm{a}, \mathrm{b})$ and of HWHM for different resonances (c, d). Solid curves in (a) and (b) are the fits by Eq. (6) with parameters: $\gamma_{0 X} / \gamma_{r}=1.1, \gamma_{0 b} / \gamma_{r}=780$ for excitation power $P=10 \mu \mathrm{W}$ in (a); $\gamma_{0 X} / \gamma_{r}=0.4, \gamma_{0 b} / \gamma_{r}=80$ for excitation power $P=150 \mu \mathrm{W}$ in (b). The activation energies for both excitation powers are: $E_{x}=4.5 \mathrm{meV}$ and $E_{b}=16 \mathrm{meV}$. The dependences were measured at the excitation into the $X 6$ transition, whose spectral position was determined at each temperature.

The value of the first activation energy obtained in the fit, $E_{X}=4.5 \mathrm{meV}$, approximately corresponds to the exciton binding energy. Therefore, we assume that the first temperature-activated process is the exciton dissociation into free carriers. The second energy, $E_{b}=16 \mathrm{meV}$, is significantly smaller than the quantization energy for excitons in the QW under study (of about $25 \mathrm{meV}$ ). It appears that the obtained value of $E_{b}$ is closer to the band offsets for free electrons and/or holes although the ratio of latter quantities is unknown and has been extensively discussed in literature up to now, see, e.g., Refs. ${ }^{52-54}$ This is why we conclude that the second temperature-activated process of PL quenching is the emission of carriers into barrier layers accompanied, possibly, by a radiative recombination in a different spectral range.

Besides the PL quenching, the temperature increase helps establishing thermal equilibrium between occupations of different exciton states. In this case, we could expect a remarkable change of the relative intensities of exciton peaks with temperature. In particular, at low temperatures, when the thermal energy, $k T$, is smaller than the energy distance between the quantum confined exciton states, the lowest exciton state should be predominantly occupied. At elevated temperatures, when $k T>E_{X 4}-E_{X 1}$, the populations of these levels should be nearly equal. The experimental data, however, show that the relative intensities of different PL lines are almost independent of temperature. This means that the efficiency of thermally activated exciton dissociation and carrier ejection is higher that the phonon-mediated tran-

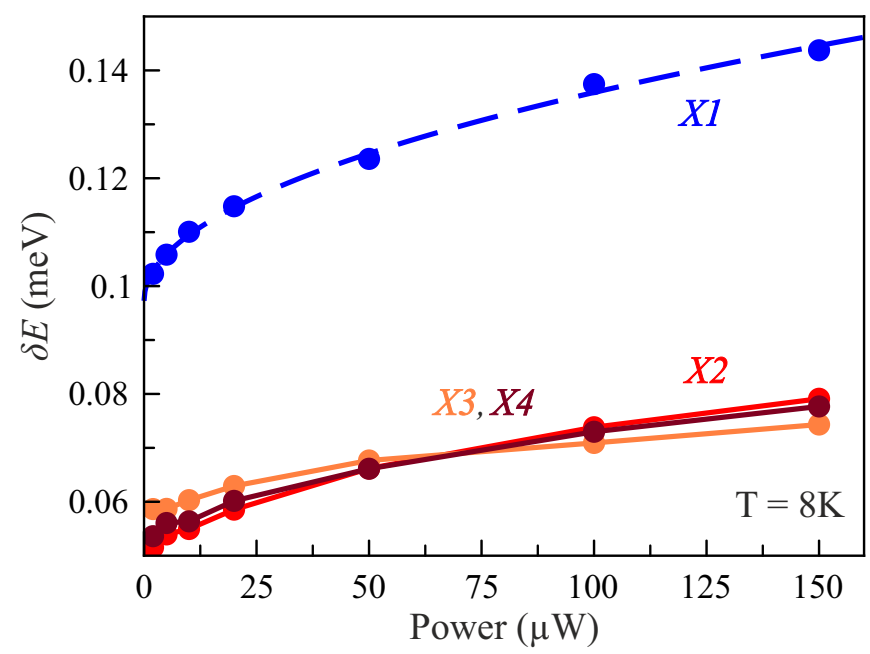

Figure 4. (Color online) Power dependences of HWHM of the resonances measured at $T=8 \mathrm{~K}$ (symbols). Curves are guides for the eye.

sitions between different exciton levels responsible for the thermal equilibrium. We conclude that the thermal equilibrium in the exciton sub-system is not achieved in this structure in the temperature range of $4-30 \mathrm{~K}$.

The widths of exciton lines also reveal a surprising, at first glance, behavior at elevated temperatures. A strong decrease of the PL yield with the temperature increase indicates that the nonradiative exciton relaxation begins to dominate over the radiative one at $T>15 \mathrm{~K}$, see Fig. 3(a, b). If the widths of exciton lines are controlled at low temperature mainly by the radiative processes, the temperature rise should result in line broadening, which is not observed in the experiment. Indeed, as seen in Fig. 3(c), the line widths measured at the low excitation power are almost constant in the temperature range studied within our experimental accuracy.

The increase of the excitation power stronger affects the line widths. Figure 4 shows that it causes an additional line broadening, which is approximately proportional to the square root of the pump power. The additional broadening is a nonmonotonic function of temperature: it increases with the temperature rise up to, approximately, $T=15 \mathrm{~K}$ and then falls down with the further sample heating [comp. Figs 3(c) and (d)]. This behavior is observed for all the excitation powers we used where the additional broadening can be reliably identified.

\section{Kinetics of exciton states}

The most direct knowledge of the exciton relaxation processes can be obtained in kinetic experiments. We have performed pump-probe experiments in the reflection geometry. Figure 5(a) shows the differential reflectance signal for the lowest exciton transition as a function of 

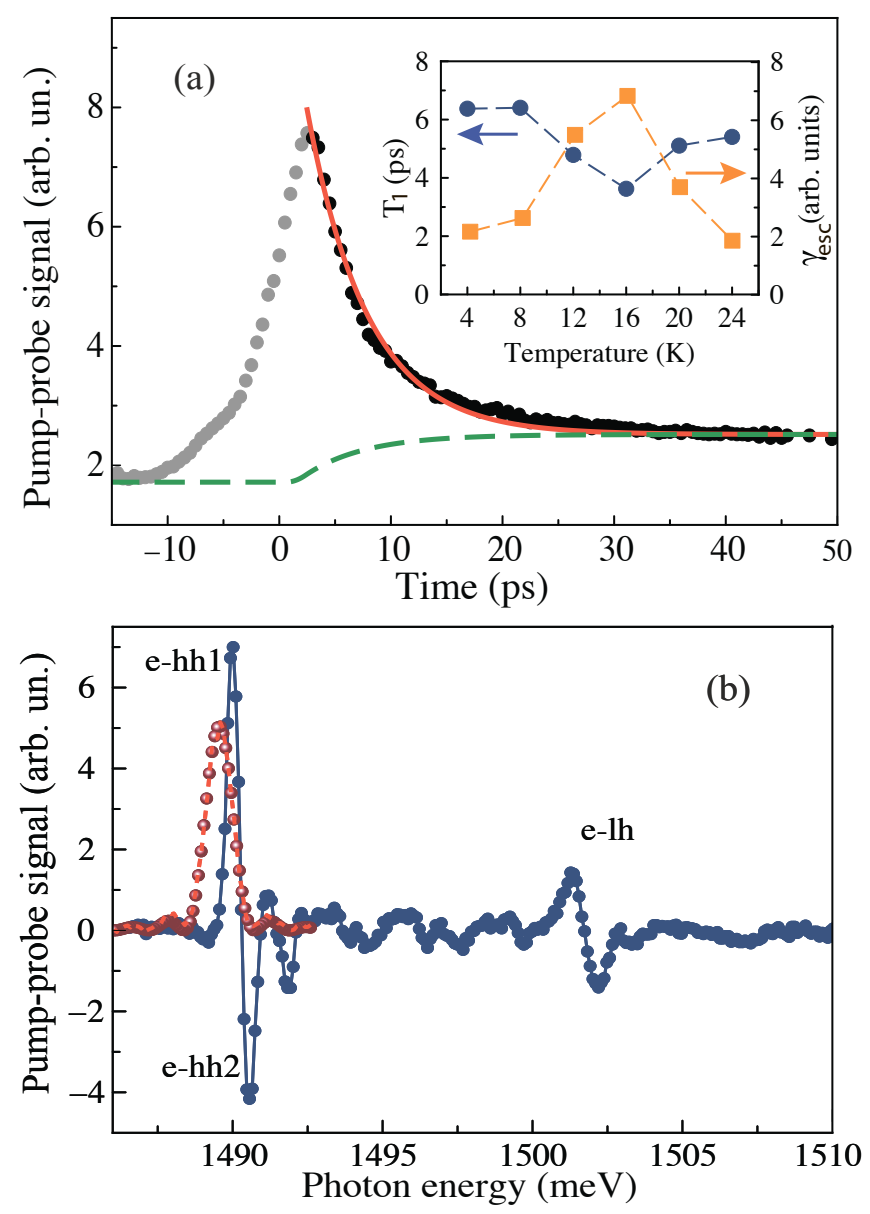

Figure 5. (Color online) (a) Kinetics of pump-probe signal measured at the $X 1$ resonance at $T=4 \mathrm{~K}$ and pump power $P_{p u}=10 \mu \mathrm{W}$ (dots). The smooth solid curve shows the fit of the fast component decay by function $f_{\text {fast }}(t)$ [see Eq. (11)]. The dashed curve shows the rise of slow component modeled by Eq. (10). Inset shows temperature dependences of $T_{1}$ (blue dots) and $\gamma_{e s c}$ (orange squares). (b) Spectral dependence of differential reflection measured at the 30-ps delay after the pump pulse (blue curve). Dotted red curve shows the spectrum of the pump pulse. Dashed curve is a modeling of the pulse spectrum by function $I_{p u}(\omega)$ given by Eq. (12) with $\hbar \delta \omega=0.37 \mathrm{meV}$.

delay between pump and probe pulses. An important peculiarity of the signal is the simultaneous presence of fast and slow components. The slow component is almost unchanged during a few hundreds of picoseconds. The fast component of kinetics reflects the dynamics of radiative excitons. In general case, it can be formed by the coherent and incoherent contributions. The coherent part of the signal is due to the four-wave mixing process. ${ }^{55} \mathrm{It}$ forms the leading edge of fast component when the probe pulse precedes the pump one, $t_{p r}<t_{p u}$. The analysis of coherent signal in detail is out of scope of present work.

The falling edge of fast component is formed by an ordinary pump-probe signal, which is determined by the exciton population created by the pump pulse. Assuming that the pump pulse has a finite duration $\left(T_{p u}=1.75 \mathrm{ps}\right.$, see Sect. I), and the probe pulse is very short ( $\delta$-pulse), we obtain following expression for the decaying part of signal:

$$
f_{p p}\left(t_{p r}\right)=I_{p r} \int I_{p u}(t) \theta\left(t_{p r}-t\right) \exp \left(-\frac{t_{p r}-t}{T_{1}}\right) d t .
$$

Here we integrate over pump pulse and take into account the sequence of pulses introducing the step-like $\theta$ function. Quantities $I_{p u}(t)$ and $I_{p r}$ are the intensities of the pump and probe beams. The pump-probe signal is proportional to the first powers of the pump and probe intensities and its dependence on the time delay is determined by the decay time of exciton population, $T_{1}$.

The fast component is superimposed on the slow one caused by the nonradiative excitons. It arises due to the ejection of radiative excitons beyond the light cone. Therefore the rise of this component is also fast and modeled as:

$$
f_{\text {slow }}\left(t_{p r}\right)=\alpha_{n r} \gamma_{e s c} \int_{0}^{t_{p r}} f_{p p}(t) d t
$$

where $\gamma_{e s c}$ is the rate of exciton ejection and constant $\alpha_{n r} \leq 1$ takes into account that the contribution of nonradiative excitons into the pump-probe signal may be smaller than that of radiative ones for the same exciton density.

The total signal detected in the experiment is the sum of both the contributions:

$$
f_{\text {fast }}\left(t_{p r}\right)=f_{p p}\left(t_{p r}\right)+f_{\text {slow }}\left(t_{p r}\right) .
$$

In the modeling of pump-probe signal, we assume that the pump pulse has a rectangular temporal profile with duration $T_{p u}$. Its spectrum shown in Fig. 5(b) can be well fitted by function:

$$
I_{p u}(\omega)=I_{p u 0}\left(\frac{\sin \left[\left(\omega-\omega_{0}\right) / \delta \omega\right]}{\left(\omega-\omega_{0}\right) / \delta \omega}\right)^{2} .
$$

This function is obtained by the Fourier transformation of a rectangular optical pulse with carrier optical frequency $\omega_{0}$ and duration $T_{p u}=1 / \delta \omega$.

Figure 5(a) shows the fit of falling edge of the fast component by function $f_{\text {fast }}(t)$. The rise of slow component is also shown by a separate curve. One can see that the decay of fast component is well modeled by function $f_{\text {fast }}(t)$ that allows us to obtain decay time $T_{1}$ of exciton population. For temperature $T=4 \mathrm{~K}$, time $T_{1}=6.4 \mathrm{ps}$, which is close to that obtained from the reflectance spectrum, $1 / \Gamma_{01}=6.9 \mathrm{ps}$.

The inset in this figure shows the temperature dependence of $T_{1}$. As seen, it strongly decreases when the temperature increases up to $16 \mathrm{~K}$ and then rises again. To analyze this effect, we assume that the decay of fast component is determined by the two main processes, the radiative decay and the exciton ejection characterized by rate $\gamma_{e s c}$. Correspondingly, the decay rate is:

$$
1 / T_{1}=\Gamma_{01}+\gamma_{e s c} .
$$




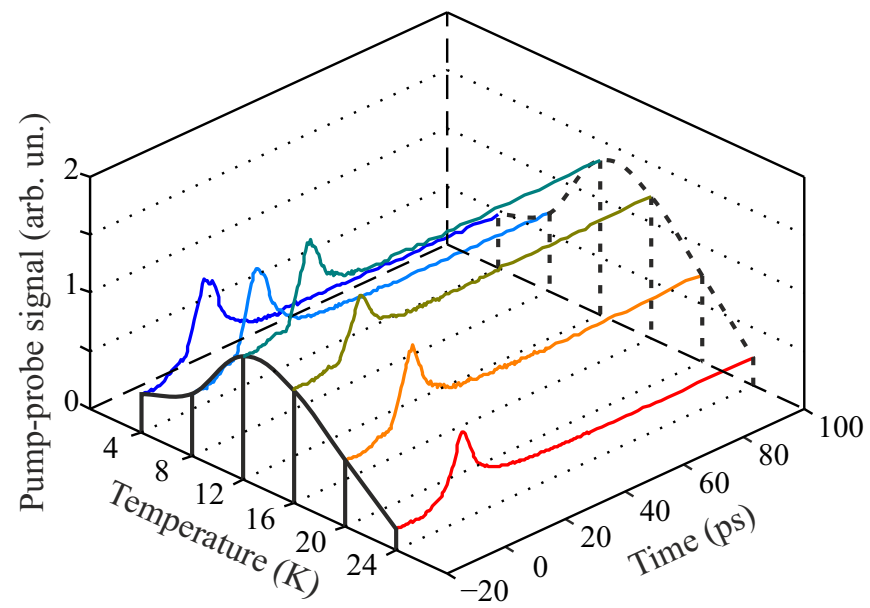

Figure 6. (Color online) Temperature variations of the pumpprobe signal for the lowest exciton transition. $P_{\text {exc }}=100 \mu \mathrm{W}$.

The temperature dependence of $\gamma_{e s c}$ can be obtained from experimental data. Indeed, the amplitude of slow component at time $t>>T_{p u}$ becomes independent of time and is determined by $\gamma_{e s c}$. From Eq. (10)] we obtain:

$$
\Delta f_{\text {slow }}=\frac{f_{\text {slow }}}{f_{p p}\left(T_{p u}\right)}=\alpha_{n r} \gamma_{e s c} T_{1} e^{T_{p u} / T_{1}}
$$

Here we normalized the amplitude of slow component on the peak amplitude of pump-probe signal described by Eq. (9) to exclude intensities of the pump and probe beams. The temperature dependence of $\gamma_{\text {esc }}$ is shown in the inset of Fig. 5(a). As seen this rate strongly increases at $T=16 \mathrm{~K}$ and then drops again. Such behavior cannot be explained by the exciton-phonon scattering because the rate of this scattering should monotonically depend on temperature in the range under study (see, e.g., Ref. ${ }^{45}$ ). Possible physical mechanism of the exciton ejection will be discussed in the next section.

Figure 5(b) shows the spectral dependence of differential reflection measured at the time delay $30 \mathrm{ps}$ when only the slow component persists. The pump pulses were spectrally narrow as shown in the figure. Although the spectral position of the pump pulses has been chosen to excite predominantly the lowest exciton level, the nonzero signal is observed in the wide spectral range up to the light-hole $\left(X_{l h}\right)$ exciton transition.

Temperature variations of the pump-probe signal are shown in Fig. 6. One can see that a considerable signal appears at the negative delay where the probe pulse tests the sample before the pump pulse arrives. The temperature rise from $4 \mathrm{~K}$ to $12 \mathrm{~K}$ causes a considerable rise of this signal, which then drops at further increase of the temperature.

The presence of the pump-probe signal at negative delays indicates that the characteristic relaxation time of the slow component, $\tau_{\text {slow }}$, exceeds the repetition period of excitation pulses $\left(T_{l}=12.5 \mathrm{~ns}\right)$. As a result, the detected signal is accumulated from many preceding pump pulses. In the case of exponential decay of the signal, the amplitude of the slow component created by a single pulse becomes just before coming the next pulse equal to: $I_{p p 1}=f_{\text {slow }} \exp \left(-T_{l} / \tau_{\text {slow }}\right)$. The total signal for all the preceding pulses can be easily calculated as a sum of the geometric progression: $I_{p p}=$ $f_{\text {slow }} \exp \left(-T_{l} / \tau_{\text {slow }}\right) /\left[1-\exp \left(-T_{l} / \tau_{\text {slow }}\right)\right]$. This expression allows us to roughly estimate the relaxation time $\tau_{\text {slow }}$ using the experimentally measured ratio $f_{\text {slow }} / I_{p p}$. Time $\tau_{\text {slow }}$ increases from $15 \mathrm{~ns}$ to $45 \mathrm{~ns}$ in the temperature range of $4-12 \mathrm{~K}$ and rapidly drops with the further temperature increase. At $T>24 \mathrm{~K}$, the signal at negative delays is not detectable. This means that the decay time $\tau_{\text {slow }}$ is shorter than the pulse repetition period $T_{l}$.

\section{DISCUSSION}

The experimental results presented above clearly show that, at low temperature and low excitation power, the structure under study is characterized by extremely narrow widths of exciton resonances and demonstrate zero Stokes shifts between the resonances observed in PL and reflectance spectra. This is a clear indication of the absence of noticeable inhomogeneous broadening of spectral lines. The line width is controlled solely by the relaxation processes.

The absence of any noticeable broadening of the resonances with the temperature rise at quasiresonant excitation with low power (see Fig. 3) means that the relaxation of radiative excitons is not affected by the excitonphonon scattering in the temperature range studied. At the same time, the peak width remarkably rises with excitation power (Fig. 4). Because the pump power increase leads to the increase of the exciton density, the observed additional broadening, $\delta E_{c}$, is most probably related to exciton-exciton collisions resulting in the relaxation of exciton states. The rate of relaxation should be proportional to the exciton density and, therefore, to the excitation power. The experiment, however, shows a sublinear power dependence, see Fig. 4. We have to assume that the nonlinearity is caused by the collision-induced decrease of the radiative recombination rate and of the corresponding broadening, $\hbar \Gamma_{0}$, due to the decrease of the exciton coherence volume. Such effect of additional relaxation on the radiative recombination rate of excitons is theoretically discussed in Refs. ${ }^{2,15,26}$ and experimentally studied in Refs. ${ }^{5,33,59}$ The decrease of radiative broadening partially compensates the collision-induced broadening so that the total peak broadening, $\delta E=\hbar \Gamma_{0}+\delta E_{c}$, should depend on power sublinearly. ${ }^{26}$ The linear dependence of integral intensities of exciton peaks on the excitation power (not shown here) evidences that there is no noticeable contribution of other mechanisms, e.g., of nonradiative exciton recombination, to the line broadening in these experimental conditions.

The above-noted increase of relative intensity and 
width of the first exciton peak at high photon energy of excitation (see Fig. 2) requires a particular attention. These observations point out that the cascade relaxation of photocreated excitons over the quantum confined states is replaced by the direct carrier relaxation into the lowest exciton level. Indeed, at the excitation above the $X_{l h}$ transition, the probability of resonant excitation with generation of excitons becomes smaller than that of generation of free carriers. The photocreated electrons and holes relax to their ground states where they are bound in excitons. The excitons thus created populate predominantly the lowest energy state, that explains the increased intensity of the $X 1$ peak at these excitation conditions. Our assumption about the photocreation of free carriers is further supported by the observation of a strong broadening of the lowest exciton peak. Indeed, according to Refs. ${ }^{23,33}$ the cross-section of exciton-freecarrier scattering is by an order of magnitude larger than that of the exciton-exciton one. So, the exciton relaxation due to exciton-carrier collisions is mainly responsible for the strong broadening of the $X 1$ resonance in the case of excitation above the $X_{l h}$ transition.

The temperature dependence of the PL peak intensities and widths (see Fig. 3) appears to be contradictory at the first glance. The temperature increase induces a remarkable decrease of the integral PL that points out to the activation of efficient nonradiative relaxation processes for excitons. The relaxation would seem to be accompanied by a noticeable broadening of exciton peaks, which is not observed in the experiment.

The origin of this effect is related to the temperature dependence of the exciton density in the reservoir. The nonradiative excitons can be efficiently created under nonresonant excitation via one-phonon relaxation as schematically shown in Fig. 7. According to the selection rule, the exciton wave vector in the QW plane should to be equal to the projection of the phonon wave vector onto the plane. The energy difference between the nearest exciton levels in our structure is about $0.5 \mathrm{meV}$ or larger. We can estimate the wave vector acquired by an exciton in the course of relaxation via emission of a longitudinal acoustic (LA) phonon. Note that the LA phonons stronger interact with excitons than other acoustic phonons. ${ }^{13,25,27}$ The wave vector of a LA phonon of energy about $0.5 \mathrm{meV}$ in GaAs is $0.07 \mathrm{~nm}^{-1}$, approximately. The light cone is limited by the photon wave vector, $K_{c} \approx 0.03 \mathrm{~nm}^{-1}$ in GaAs, that is smaller than the phonon wave vector. So, the most part of excitons created by a nonresonant optical excitation should be nonradiative ones.

At low temperature, the main decay channel of nonradiative excitons in high-quality structure is the wave vector relaxation schematically shown in Fig. 7. As a result, nonradiative excitons convert into radiative ones and eventually recombine. The rate of this relaxation should be very limited because the radiative states are just a small fraction of all states within the exciton band, and the probability for an exciton to be found in one of

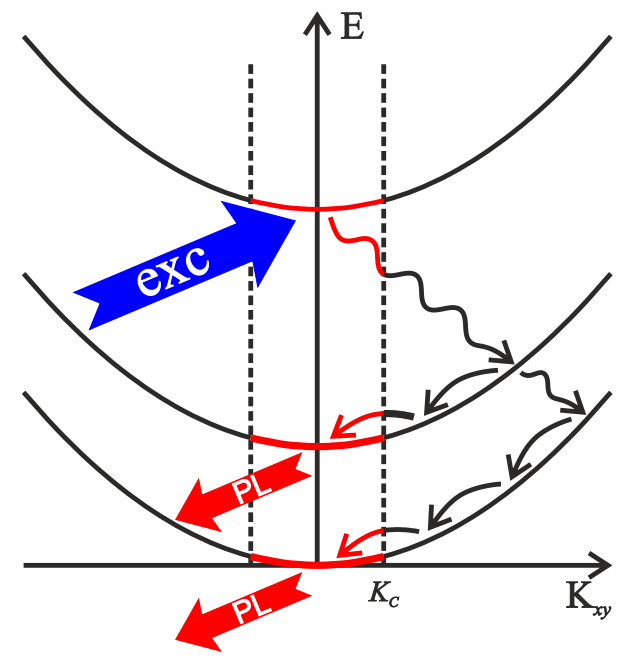

Figure 7. (Color online) A simplified sketch of the processes discussed. Parabolas show the dispersion of the quantum confined exciton states along the QW layer. Vertical dashed lines restrict the light cone area of wave vectors. The bold arrows show excitation and PL processes and the wavy arrows show the phonon-mediated exciton relaxation.

the radiative states is low. This is why, the lifetime of nonradiative excitons may drastically exceed that of radiative one.

We believe that nonradiative excitons are responsible for the long-lived component of signal in pump-probe experiments, shown in Figs. 5 and 6. In these experiments, the reservoir of nonradiative excitons can be filled in by ejection of radiative excitons as it is discussed in the preceding section. Besides, due to the finite spectral width of the pump pulses as well as the presence of some spectral wings [see Fig. 5(b)], the excitation of the high-energy exciton transitions is also probable. Exciton relaxation from the excited states populates the reservoir of nonradiative excitons. The lifetime of the long-lived component, $\tau_{\text {slow }}$, characterizes the rate of transformation of the nonradiative excitons into radiative ones. At low temperature $\tau_{\text {slow }} \approx 15 \mathrm{~ns}$, which is orders of magnitude longer than the lifetime of radiative excitons.

Nonradiative excitons do not directly interact with light. However, as we can see, they give some indirect contribution to reflectance. Because of orthogonality of the wave functions of excitons with different wave vectors, their contribution to the pump-probe signal should not been connected with bleaching of exciton transitions due to phase-space filling considered in Refs. ${ }^{56-58}$ The Coulomb screening effect discussed in these papers is not expected to play a major role at low excitation powers used in our experiments. More probably, the experimentally observed changes in differential reflection are due to the scattering of radiative excitons by nonradiative ones. The scattering results in the broadening of exciton transitions, which is observed, particularly, in the PL spectra at strong pumping, see Fig. 3(d). The broadening 
changes the reflectivity, which is detected as the pumpprobe signal and, correspondingly, as the change of the differential reflectance in the vicinity of high-energy exciton transitions, observed experimentally, see Fig. 5(b).

The huge difference in lifetimes of the radiative and nonradiative excitons explains the contradictory temperature behavior of the PL peaks mentioned above. Indeed, the radiative states are mainly populated by the cascade relaxation of photocreated excitons via nonradiative states. Due to the low rate of transformation of nonradiative excitons into radiative ones, almost all the excitation power is accumulated in the nonradiative exciton reservoir. In particular, the ratio of densities of nonradiative and radiative excitons, $n_{n r} / n_{r}=\tau_{\text {slow }} / T_{1} \approx 2 \times 10^{3}$ at $T=4 \mathrm{~K}$. The temperature increase triggers the exciton dissociation primarily in this reservoir. The characteristic time of this dissociation process becomes comparable to the lifetime of nonradiative excitons which leads to an efficient depopulation of the reservoir. The depopulation is evidenced by a rapid decrease of the slow component amplitude in the pump-probe signal at $T>15 \mathrm{~K}$, see Fig. 5(a). We believe that this is the process responsible for the PL quenching at elevated temperatures. At the same time, the exciton dissociation is still a slow process in comparison with the radiative recombination and, therefore, it cannot have a noticeable effect on the broadening of exciton peaks. This explains the seeming contradiction mentioned above.

The interaction of radiative excitons with nonradiative ones explains the nonmonotonic temperature dependence of the PL peak width. The increase of the width when the temperature rises up to $15 \mathrm{~K}$ is related to accumulation of nonradiative excitons, which is evidenced in the pump-probe experiments as the increase of the amplitude of long-lived component of the signal (see Fig. 6). The accumulation is caused by the increase of the average kinetic energy and, consequently, of the average wave vector of the nonradiative excitons with the temperature increase. As a result, the exciton relaxation to the light cone is slowed down and their lifetime increases.

A simple theoretical analysis predicts a linear temperature dependence of the exciton lifetime and, hence, the density of nonradiative excitons in QWs. ${ }^{4,14,51,60} \mathrm{At}$ higher temperatures, the thermo-activated dissociation of nonradiative excitons reduces their density and, consequently, diminishes the additional broadening of exciton peaks. Figure 8 shows that the peak width and the amplitude of the long lived component exhibit likewise temperature dependencies for the first exciton transition. This confirms that nonradiative excitons strongly affect both broadening and amplitude. The temperature dependence of $\delta E$ can be approximated by function:

$$
\delta E_{X 1}(T)=\delta E_{0}+\alpha T \times I(T),
$$

where $I(T)$ is given by Eq. (6) and describes the thermally activated losses of excitons in the reservoir.

The nonmonotonic temperature behavior of the exciton ejection rate, $\gamma_{e s c}$, discussed in Sect. II B is also ex-

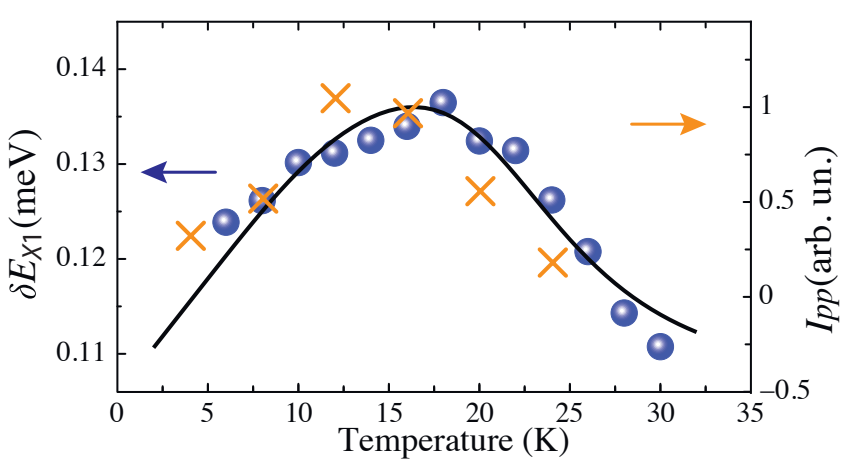

Figure 8. (Color online) Temperature variations of the line broadening $\delta E_{X 1}$ (blue balls) for transition $X 1$ measured at excitation power $P=50 \mu \mathrm{W}$ and of amplitude $I_{p p}$ (orange crosses) of the long-lived component of pump-probe signal shown in Fig. 6. Solid curve shows fit by Eq. (15) with parameters [see Eq. (6)]: $\delta E_{0}=120 \mu \mathrm{eV}, \gamma_{X 1} / \gamma_{r}=14$, $\gamma_{b} / \gamma_{r}=6000$. The activation energies are chosen the same as for PL quenching (see Fig. 3).

plained by the scattering of radiative excitons by the nonradiative ones. Rate $\gamma_{e s c}$ normalized to the amplitude $I_{p p}$ of long-lived component of the pump-probe signal (see Fig. 8) is found to be not dependent of temperature within an experimental error of about $15 \%$. Because $I_{p p}$ is proportional to the exciton density in the reservoir, $n_{n r}$, this fact means that $\gamma_{e s c} \sim n_{n r}$. We, therefore, may conclude that the ejection of radiative excitons beyond the light cone is mainly caused by their collisions with nonradiative excitons.

\section{CONCLUSION}

The analysis of PL spectra and relaxation kinetics of quantum confined exciton states in a high-quality wide QW structure allowed us to obtain a valuable information about the most important relaxation processes in this system. Extremely narrow widths of spectral lines and the absence of any Stokes shift between the resonances observed in the PL and reflectance spectra indicate high quality of the structure. Our experiments show that, because of the negligibly low density of defects, the most efficient process of exciton decay at low temperatures and low excitation powers is the radiative recombination. This process is responsible for the broadening of the low-energy exciton peak. The rate of radiative recombination for the higher energy exciton states is reduced. This is why the phonon-mediated relaxation of excitons from higher to lower energy states strongly affects the line broadening. The relaxation process is additionally favored for higher energy states since the density of acoustic phonon states increases as a function of energy.

Our study shows that the kinetics of radiative excitons is strongly affected by the long-lived reservoir of 
nonradiative excitons whose wave vectors lie outside the light cone. In particular, the reservoir manifests itself in the long-lived component of the pump-probe signal. The lifetime of this component is in three order of magnitude larger than the radiative decay time. It is governed by slow relaxation of nonradiative excitons into the radiative states. At low temperature, the slow relaxation does not lead to a reduction of the PL yield, as nonradiative recombination processes in the reservoir are inefficient in high-quality QWs. The temperature increase up to $30 \mathrm{~K}$ results in the strong decrease of exciton PL intensity with no noticeable broadening of the exciton lines. The origin of this unusual effect is in the thermal dissociation of nonradiative excitons in the reservoir. The dissociation rate is comparable to that of exciton relaxation from nonradiative to radiative states but it is drastically lower than the recombination rate of radiative excitons, which governs the widths of exciton lines. The reservoir of nonradiative excitons also affects the exciton PL spectra. In particular, the scattering of radiative excitons by nonradiative ones at high pumping intensity results in a supplementary broadening of the PL lines.

In should be emphasized that the observed nontrivial effects are not specific property of the sample under study. They can be realized in any semiconductor QWs of moderate width characterized by ultra-high quality.

\section{ACKNOWLEDGMENTS}

The authors thank M. M. Glazov and K. V. Kavokin for fruitful discussions. Financial support from the Russian Ministry of Science and Education (contract no. 11.G34.31.0067), SPbU (grants No. 11.38.213.2014) is acknowledged. The authors also thank the SPbU Resource Center "Nanophotonics" (www.photon.spbu.ru) for the sample studied in present work. A. V. Kavokin acknowledges the support from EPSRC Established Career Fellowship.
1 E. I. Rashba, G. E. Gurgenishvili, "Edge absorption theory in semiconductors", Fiz. Tverd. Tela (Leningrad) 4, 1029 (1962) [Sov. Phys. Solid State 4, 759 (1962)].

2 Eiichi Hanamura, "Rapid radiative decay and enhanced optical nonlinearity of excitons in a quantum well", Phys. Rev. B 38, 1228 (1988).

3 B. Deveaud, F. Clérot, N. Roy, K. Satzke, B. Sermage, and D. S. Katzer, "Enhanced Radiative Recombination of Free Excitons in GaAs Quantum Wells", Phys. Rev. Lett. 67, 2355 (1991).

4 L.C. Andreani, F. Tassone, F. Bassani, "Radiative lifetime of free excitons in quantum wells", Solid State Commun. 77, 641, (1991).

5 A. Vinattieri, Jagdeep Shah, T. C. Damen, D. S. Kim, L. N. Pfeiffer, M. Z. Maialle, and L. J. Sham, "Exciton dynamics in GaAs quantum wells under resonant excitation", Phys. Rev. B 50, 10868 (1994).

6 S. W. Koch, M. Kira, G. Khitrova, and H. M. Gibbs, "Semiconductor excitons in new light", Nature Mat. 5, 523 (2006).

7 D. V. Vishnevsky, D. D. Solnyshkov, N. A. Gippius, and G. Malpuech, "Multistability of cavity exciton polaritons affected by the thermally generated exciton reservoir", Phys. Rev. B 85, 155328 (2012).

8 M. Wouters, T. K. Paraïso, Y. Léger, R. Cerna, F. MorierGenoud, M. T. Portella-Oberli, and B. Deveaud-Plédran, "Influence of a nonradiative reservoir on polariton spin multistability" Phys. Rev. B 87, 045303 (2013).

9 M. De Giorgi, D. Ballarini, P. Cazzato, G. Deligeorgis, S. I. Tsintzos, Z. Hatzopoulos, P. G. Savvidis, G. Gigli, F. P. Laussy, and D. Sanvitto, "Temperature dependence of the radiative and nonradiative recombination time in GaAs $/ \mathrm{Al}_{x} \mathrm{Ga}_{1-x} \mathrm{As}$ quantum-well structures", Phys. Rev. Lett. 112, 113602 (2014).

10 S. S. Demirchyan, I. Yu. Chestnov, A. P. Alodjants, M. M. Glazov, and A. V. Kavokin, "Qubits Based on Polariton Rabi Oscillators", Phys. Rev. Lett. 112, 196403 (2014).
${ }^{11}$ H. Haug, T. D. Doan, and D. B. Tran Thoai, "Quantum kinetic derivation of the nonequilibrium Gross-Pitaevskii equation for nonresonant excitation of microcavity polaritons", Phys. Rev. B 89, 155302 (2014).

12 V. V. Belykh and D. N. Sobyanin, "Polariton linewidth and the reservoir temperature dynamics in a semiconductor microcavity", Phys. Rev. B 89, 245312 (2014).

13 C. Piermarocchi, F. Tassone, V. Savona, A. Quattropani, and P. Schwendimann, "Nonequilibrium dynamics of free quantum-well excitons in time-resolved photoluminescence", Phys. Rev. B 53, 15834 (1996).

14 J. Feldmann, G. Peter, E. O. G'obel, P. Dawson, K. Moore, C. Foxon, and R. J. Elliott, "Linewidth Dependence of Radiative Exciton Lifetimes in Quantum Wells", Phys. Rev. Lett. 59, 2337 (1987).

15 D. S. Citrin, "Radiative lifetimes of excitons in quantum wells: Localization and phase-coherence effects", Phys. Rev. B 47, 3832 (1993).

16 A. V. Kavokin, "Exciton oscillator strength in quantum wells: From localized to free resonant states", Phys. Rev. B 50, 8000 (1994).

17 T. C. Damen, Jagdeep Shah, D. Y. Oberli, D. S. Chemla, J. E. Cunningham, and J. M. Kuo, "Dynamics of exciton formation and relaxation in GaAs quantum wells", Phys. Rev. B 42, 7434 (1990).

18 Vivek Srinivas, John Hryniewicz, Yung Jui Chen, and Colin E. C. Wood, "Intrinsic linewidths and radiative lifetimes of free excitons in GaAs quantum wells", Phys. Rev. B 46, 10193 (1992).

19 J. Szczytko, L. Kappei, J. Berney, F. Morier-Genoud, M.T. Portella-Oberli, and B. Deveaud, "Determination of the Exciton Formation in Quantum Wells from Time-Resolved Interband Luminescence", Phys. Rev. Lett. 93, 137401 (2004).

20 Ph. Roussignol, C. Delalande, A. Vinattieri, L. Carraresi, and M. Colocci, "Dynamics of exciton relaxation in GaAs $/ \mathrm{Al}_{x} \mathrm{Ga}_{1-x} \mathrm{As}$ quantum wells", Phys. Rev. B 45, 6965 (1992). 
${ }^{21}$ L. Kappei, J. Szczytko, F. Morier-Genoud, and B. Deveaud, "Direct Observation of the Mott Transition in an Optically Excited Semiconductor Quantum Well", Phys. Rev. Lett. 94, 147403 (2005).

22 B. Deveaud, L. Kappei, J. Berney, F. Morier-Genoud, M.T. Portella-Oberli, J. Szczytko, and C. Piermarocchi, "Excitonic effects in the luminescence of quantum wells", Chem. Phys. 318, 104 (2005).

23 D. Bajoni, P. Senellart, M. Perrin, A. Lemaitre, B. Sermage, and J. Bloch, "Exciton dynamics in the presence of an electron gas in GaAs quantum wells", phys. stat. sol. (b) 243, 2384 (2006).

24 M. T. Portella-Oberli, J. Berney, L. Kappei, F. MorierGenoud, J. Szczytko, and B. Deveaud-Plédran, "Dynamics of Trion Formation in $\operatorname{In}_{x} \mathrm{Ga}_{1-x}$ As Quantum Wells", Phys. Rev. Lett. 102, 096402 (2009).

25 P. K. Basu and Partha Ray, "Energy relaxation of hot twodimensional excitons in a GaAs quantum well by excitonphonon interaction", Phys. Rev. B 45, 1907 (1992).

26 C. Ciuti, V. Savona, C. Piermarocchi, A. Quattropani, and P. Schwendimann, "Role of the exchange of carriers in elastic exciton-exciton scattering in quantum wells", Phys. Rev. B 58, 7926 (1998).

27 A. L. Ivanov, P. B. Littlewood, and H. Haug, "BoseEinstein statistics in thermalization and photoluminescence of quantum-well excitons", Phys. Rev. B 59, 5032 (1999).

28 C. Piermarocchi, V. Savona, A. Quattropani, P. Schwendimann, and F. Tassone, "Photoluminescence and Carrier Dynamics in GaAs Quantum Wells", phys. stat. sol. (a) 164, 221 (1997).

29 M. Gurioli, A. Vinattieri, M. Colocci, C. Deparis, J. Massies, G. Neu, A. Bosacchi, and S. Franchi, "Temperature dependence of the radiative and nonradiative recombination time in $\mathrm{GaAs} / \mathrm{Al}_{x} \mathrm{Ga}_{1-x}$ As quantum-well structures", Phys. Rev. B 44, 3115 (1991).

${ }^{30}$ R. Eccleston, B. F. Feuerbacher, J. Kuhl, W. W. Rühle, and K. Ploog, "Density-dependent exciton radiative lifetimes in GaAs quantum wells", Phys. Rev. B 45, 11403 (1992).

31 G. Finkelstein, V. Umansky, I. Bar-Joseph, V. Ciulin, S. Haacke, J.-D. Ganiére, and B. Deveaud, "Charged exciton dynamics in GaAs quantum wells", Phys. Rev. B 58, 12637 (1998).

32 L. Schultheis, A. Honold, J. Kuhl, K. Köhler, and C. W. $\mathrm{Tu}$, "Optical dephasing of homogeneously broadened twodimensional exciton transitions in GaAs quantum wells", Phys. Rev. B 34, 9027 (1986).

33 A. Honold, L. Schultheis, J. Kuhl, and C. W. Tu, "Collision broadening of two-dimensional excitons in a GaAs single quantum well", Phys. Rev. B 40, 6442 (1989).

${ }^{34}$ Dai-Sik Kim, Jagdeep Shah, T. C. Damen, W. Schäfer, F. Jahnke, S. Schmitt-Rink, and K. Köhler, "Unusually Slow Temporal Evolution of Femtosecond Four-Wave-Mixing Signals in Intrinsic GaAs Quantum Wells: Direct Evidence for the Dominance of Interaction Effects", Phys. Rev. Lett. 69, 2725 (1992).

35 R. Duer, I. Shtrichman, D. Gershoni, and E. Ehrenfreund, "Momentum Redistribution Times of Resonantly Photogenerated 2D Excitons", Phys. Rev. Lett. 78, 3919 (1997).

36 P. Borri, W. Langbein, J. M. Hvam, and F. Martelli, "Well-width dependence of exciton-phonon scattering in $\mathrm{In}_{x} \mathrm{Ga}_{1-x} \mathrm{As} / \mathrm{GaAs}$ single quantum wells", Phys. Rev. B 59, 2215 (1999).
37 D. S. Chemla and Jagdeep Shah, "Many-body and correlation effects in semiconductors", Nature 411, 549 (2001).

38 R. P. Smith, J. K. Wahlstrand, A. C. Funk, R. P. Mirin, S. T. Cundiff, J. T. Steiner, M. Schafer, M. Kira, and S.W. Koch, "Extraction of Many-Body Configurations from Nonlinear Absorption in Semiconductor Quantum Wells", Phys. Rev. Lett. 104, 247401 (2010).

39 A. Uddin and T. G. Andersson, "Investigation of highquality GaAs:In layers grown by molecular-beam epitaxy", J. Appl. Phys. 65, 3101 (1989).

40 E. L. Ivchenko, "Optical spectroscopy of semiconductor nanostructures", Springer (Berlin) 2004, 437 p.

41 E. V. Ubyivovk, D. K. Loginov, I. Ya. Gerlovin, Yu. K. Dolgikh, Yu. P. Efimov, S. A. Eliseev, V. V. Petrov, O. F. Vyvenko, A. A. Sitnikova, and D. A. Kirilenko, "Experimental determination of dead layer thickness for excitons in a wide GaAs/AlGaAs quantum well", Fiz. Tv. Tela 51, 1818 (2009) [Phys. Sol. State 51, 1929 (2009)].

42 D. Schiumarini, N. Tomassini, L. Pilozzi, and A. D'Andrea, "Polariton propagation in weak-confinement quantum wells', Phys. Rev. B 82, 075303 (2010).

43 A. Tredicucci, Y. Chen, F. Bassani, J. Massies, C. Deparis, G. Neu, "Center-of-mass quantization of excitons and polariton interference in GaAs thin layers", Phys. Rev. B 47, 10348 (1993).

${ }^{44}$ K. Muraki, S. Fukatsu, and Y. Shiraki, "Surface segregation of In atoms during molecular beam epitaxy and its influence on the energy levels in InGaAs/GaAs quantum wells", Appl. Phys. Lett. 61, 557 (1992).

45 S.V. Poltavtsev, Yu.P. Efimov, Yu.K. Dolgikh, S.A. Eliseev, V.V. Petrov, V.V. Ovsyankin, "Extremely low inhomogeneous broadening of exciton lines in shallow (In,Ga)As/GaAs quantum wells", Solid State Commun. 199, 47 (2014)

46 Y.J. Chen, Emil S. Koteles, Johnson Lee, J.Y. Chi, B.S. Elman, "Temperature dependent optical studies of GaAs/AlGaAs single quantum wells", SPIE 792, 162 (1987).

47 Rohan Singh, Travis M. Autry, Gaël Nardin, Galan Moody, Hebin Li, Klaus Pierz, Mark Bieler, and Steven T. Cundiff, "Anisotropic homogeneous linewidth of the heavy-hole exciton in (110)-oriented GaAs quantum wells", Phys. Rev. B 88, 045304 (2013).

48 H. Tuffigo, R. T. Cox, N. Magnea, Y. Merle d'Aubigné, and A. Million, "Luminescence from quantized excitonpolariton states in $\mathrm{Cd}_{1-x} \mathrm{Zn}_{x} \mathrm{Te} / \mathrm{CdTe} / \mathrm{Cd}_{1-x} \mathrm{Zn}_{x}$ Te thinlayer heterostructures", Phys. Rev. B 37, 4310 (1988).

49 The low magnitude quasiperiodic oscillations of the PL intensity are due to the interference of the excitation laser light reflected from the front and rear surfaces of the sample.

50 E.L. Ivchenko, G.E. Pikus, B.S. Razbirin, A.I. Starukhin, "Optical orientation and alignment of free excitons in GaSe during resonant excitation - Theory", Russian ZhETF $\mathbf{7 2}$, 2230 (1977) [JETP 45, 1172 (1977)].

51 D. Rosales, T. Bretagnon, B. Gil, A. Kahouli, J. Brault, B. Damilano, J. Massies, M. V. Durnev, and A. V. Kavokin, "Excitons in nitride heterostructures: From zero- to onedimensional behavior", Phys. Rev. B 88, 125437(2013).

${ }^{52}$ M. J. Joyce, Z. Y. Xu, and M. Gal, "Photoluminescence excitation spectroscopy of as-grown and chemically released $\mathrm{In}_{0} .05 \mathrm{Ga}_{0} .95 \mathrm{As} / \mathrm{GaAs}$ quantum wells", Phys. Rev. B 44, 3144 (1991). 
53 V. I. Zubkov, M. A. Melnik, A. V. Solomonov, and E. O. Tsvelev, F. Bugge, M. Weyers, and G. Tränkle, "Determination of band offsets in strained $\operatorname{In}_{x} \mathrm{Ga}_{1-x} \mathrm{As} / \mathrm{GaAs}$ quantum wells by capacitance-voltage profiling and Schr'odinger-Poisson self-consistent simulation", Phys. Rev. B 70, 075312 (2004).

54 Dipankar Biswas, Partha Pratim Bera, Tapas Das, Siddhartha Panda, "Impact of strain on the band offsets of important III-V quantum wells: $\operatorname{In}_{x} \mathrm{Ga}_{1-x} \mathrm{~N} / \mathrm{GaN}$, GaAs $/ \operatorname{In}_{x} \mathrm{Ga}_{1-x} \mathrm{P}$ and $\operatorname{In}_{x} \mathrm{Ga}_{1-x}$ As/AlGaAs", Materials Sci. Semicond. Processing 19, 11 (2014).

55 J. Shah, "Ultrafast spectroscopy of semiconductor and semiconductor nanostructures", Berlin, "Springer-Verlag", 1996, 372 pp.

${ }^{56}$ S. Schmitt-Rink, D. S. Chemla, and D. A. B. Miller, "Theory of transient excitonic optical nonlinearities in semiconductor quantum-well structures", Phys. Rev. B 32, 6601 (1985).
57 S. Hunsche, K. Leo, H. Kurz, and K. Köhler, "Exciton absorption saturation by phase-space filling: Influence of carrier temperature and density", Phys. Rev. B 49, 16565 (1994).

58 M. Koch, J. Feldmann, E.O. Göbel, P. Thomas, and J. Shah, and K. Köhler, "Coupling of exciton transitions associated with different quantum-well islands", Phys. Rev. B 48, 11480 (1993).

59 Félix Fernández-Alonso, Marcofabio Righini, Andrea Franco, and Stefano Selci, "Time-resolved differential reflectivity as a probe of on-resonance exciton dynamics in quantum wells", Phys. Rev. B 67, 165328 (2003).

60 In our case of relatively wide QW, the density of states of nonradiative excitons is a step function with steps at the each quantum confined state. Therefore, the temperature dependence of the exciton lifetime deviates from the linear one proposed for two dimensional excitons and tends to approach the $T^{3 / 2}$ dependence characteristic of the bulk material, see, e.g., Ref.. ${ }^{51}$ We use the linear dependence in the fit in Fig. 8 in order to minimize the number of fitting parameters. 